\title{
Effects of Eccentric Contractions Induced Electrical Stimulation Training System on Quadriceps Femoris Muscle
}

\author{
Minoru Tanaka1,2, Ryosuke Nakanishi' ${ }^{1}$, Noriaki Maeshige1, Hidemi Fujino ${ }^{1 *}$ \\ ${ }^{1}$ Department of Rehabilitation Science, Graduate School of Health Sciences, Kobe University, Kobe, Japan \\ ${ }^{2}$ Faculty of Health Science, Department of Physical Therapy, Osaka Yukioka College of Health Science, Osaka, Japan \\ Email: *fujino@phoenix.kobe-u.ac.jp
}

How to cite this paper: Tanaka, M., Nakanishi, R., Maeshige, N. and Fujino, H. (2017) Effects of Eccentric Contractions Induced Electrical Stimulation Training System on Quadriceps Femoris Muscle. International Journal of Clinical Medicine, 8, 519-533.

https://doi.org/10.4236/ijcm.2017.89049

Received: August 7, 2017

Accepted: September 24, 2017

Published: September 27, 2017

Copyright $\odot 2017$ by authors and Scientific Research Publishing Inc. This work is licensed under the Creative Commons Attribution International License (CC BY 4.0).

http://creativecommons.org/licenses/by/4.0/

(c) (i) Open Access

\begin{abstract}
We developed an eccentric contraction induced electrical stimulation (ES) training system. The purpose of this study was to investigate whether the eccentric contraction induced ES enhance the knee extension torque compared with typical ES. Twenty-two young untrained men (age: $23 \pm 3$ years) in the acute response trial (single training) and seven untrained men in the long period training trial (for 6 weeks) were studied. We measured muscle thickness and knee extension torque evoked by ES with eccentric contraction training system (ES + ECC) or ES alone for the quadriceps muscle of men. The levels of pain and discomfort were evaluated using numeric rating scale (NRS) and heart rate variability. The knee extension torque of ES + ECC was higher than that of ES alone in the acute response trial. There were no significant differences in the levels of pain and discomfort between ES and ES + ECC. Additionally, ES + ECC training for 6 weeks was effective on the quadriceps muscle thickness and knee extension torque. In contrast, the ES alone training failed to increase muscle thickness and knee extension torque. These results suggest that eccentric contraction induced ES would have the potential to become an effective intervention to promote muscle strengthening.
\end{abstract}

\section{Keywords}

Eccentric Contraction, Muscle Strengthening, Neuromuscular Electrical Stimulation, Middle Frequency

\section{Introduction}

Resistance exercise can be effective for muscle strengthening [1]. The effect of 
resistance exercise is known to be dependent on the intensity of muscle loading [2]. Exercise involving eccentric contractions has a greater effect for muscle strengthening because the high intensity of muscle loading can be generated eccentric contraction compared to concentric or isometric contractions [3] [4]. The previous studies have suggested that eccentric exercise has advantages compared with concentric training, e.g., increases in peak torque and strength-related performance parameters [5] [6]. Therefore, eccentric exercise might have an efficient exercise for muscle strengthening compared to concentric or isometric exercise.

It has been well established that electrical stimulation (ES) can be effective to induce muscle strengthening [7] [8] [9] [10]. The effectiveness of ES is determined by the intensity of muscle loading, as well as resistance exercise [11] [12] [13]. The electrical stimulation-induced muscle loading is influenced by the current intensity, current frequency, and waveform [12] [14]. ES with low frequency direct current is commonly used in electrical stimulation therapy [14]. However, it has been suggested that ES with low frequency direct current cannot elicit muscle contraction in the deep portion of the limb due to its low conductivity [11]. Slow fiber muscles locate in the deep portion of the extremities and the trunk mainly, and fast muscles locate in the superficial portion [15]. Deep portion of muscles have an important muscle function, e.g., joint stability and maintaining posture [16]. Our previous study suggested that middle frequency electrical stimulation could induce strong contraction to skeletal muscle located deep portion of calf muscles compared with low frequency electrical stimulation [13]. Therefore, ES with middle frequency has a potential to be the effective intervention for deep muscle strengthening.

In contrast, ES causes pain and discomfort [17]. Additionally, the levels of pain and discomfort by ES depend on current intensity [17]. Therefore, the intensity of ES could not increase for strong muscle contraction and it is necessary to develop new methods for muscle strengthening without pain in the deep portion of the extremities. As a solution of problem with ES for muscle strengthening, it has reported that ES combined voluntary eccentric contraction which an agonist performs a voluntary concentric contraction against an electrically stimulated antagonist was developed [18]. However, this eccentric contraction training has some limitations [19]. The patients who have severely affected with neuromuscular diseases might not be adequate the eccentric contraction because they need to be able to generate agonist muscle forces to overcome the resistance provided by the electrically stimulated antagonist. Additionally, this eccentric contraction could not set the joint range and maintain a constant angular velocity to the joint. To improve those problems, we developed an ES with eccentric contraction system. The purpose of this study was to investigate the acute response whether eccentric contraction induced electrical stimulation training system enhance muscle knee extension torque compared with typical electrical stimulation method and to evaluate the long period training the effects. 


\section{Materials and Methods}

\subsection{Participants}

This study recruited twenty-two young untrained men (mean age \pm SD: $23 \pm 3$ years, height: $176 \pm 7 \mathrm{~cm}$, mass: $67 \pm 7 \mathrm{~kg}$, respectively) who responded to an invitation to participate in the acute response trial (Experiment 1) and seven young untrained men (mean age \pm SD: $23 \pm 8$ years, height: $175 \pm 9 \mathrm{~cm}$, mass: 67 $\pm 3 \mathrm{~kg}$, respectively) who responded to an additional invitation to participate in 6-weeks training trial (Experiment 2). In the acute response trial, the subjects were measured in the left limb. In the long period training trial, the subjects were trained the both limbs. The subjects were free from known cardiovascular, neurological, or orthopedic problems, volunteered to participate in the study. The subjects were asked to avoid stimulants (e.g. alcohol, caffeine, chocolate) and exercise on the test day, and did not perform any intense exercise 2 days prior to the tests. The subjects were informed of all the procedures, purposes, benefits, and risks of the study and signed an informed consent form, which was approved by the Medical Ethical Committee of Kobe University in accordance with the Declaration of Helsinki. We measured Experiment 1 from February in 2015 to April in 2015 and Experiment 2 from July in 2015 to September in 2015.

\subsection{Electrical Stimulation with Eccentric Contraction System}

Our eccentric contraction induced electrical stimulation system consists of two parts: 1 ) a continuous passive movement (CPM) device for the knee joint; and 2) a ES device with controller. The CPM device includes an actuator (EASM6, oriental motor, Tokyo, Japan) to generate knee movements with a set velocity which can set freely and an exoskeleton to fix the limb. The exoskeleton was designed to allow the knee joint ROM from $5^{\circ}$ (fully extended) to $100^{\circ}$ (flexed). The ES device (ES-360, Ito, Tokyo, Japan) was used to stimulate the quadriceps femoris muscle focus on vastus intermedius (VI) muscle only while the knee joint was flexing; thus, VI muscle could perform eccentric contractions without voluntary contraction. A controller was used to link the CPM and the ES device, controlling the knee joint movement using the current intensity modulation function and triggering the knee joint was in flexion only while (Figure 1). During the training, the subject was required to maintain supine position and the start position with hip and knee joint angles were fixed at $30^{\circ}$ and $5^{\circ}$, respectively.

\subsection{ES Procedures}

The effects in the acute response trial compared between before and after exercise session (a single bout training) in Experiment 1. In addition, the long period training trial compared between before the first training and after $48 \mathrm{~h}$ from the last training day in Experiment 2. One burst of electrical stimulation was delivered every $3 \mathrm{sec}$ (time on: $1 \mathrm{sec}$ and time off: $2 \mathrm{sec}$ ) for $1 \mathrm{~min}$, followed by $5 \mathrm{~min}$ of rest. Exercise which included six consecutive stimulation sessions was performed. 


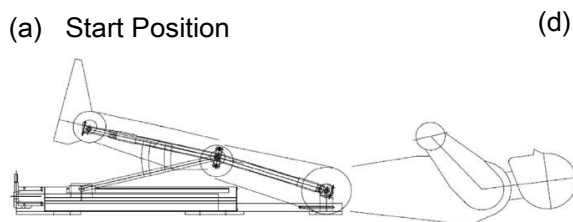

End Position

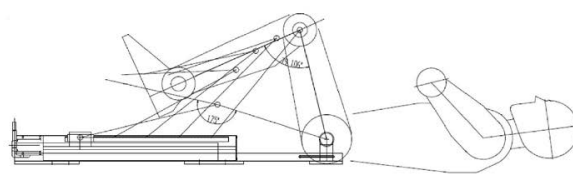

(b)

Electrical stimulator

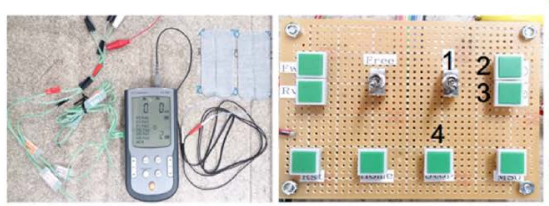

(C)

Controller

(d)
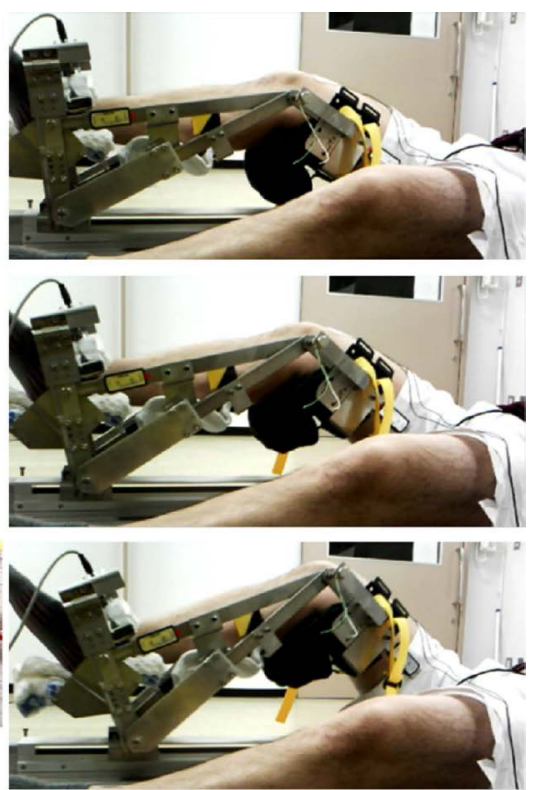

Figure 1. Apparatus for eccentric contraction induced ES exercise and its application. (a) Schema of moving during ES exercise; (b) Electrical stimulator using ES; (c) Actuator controller (1: start, 2: current up, 3: current down, 4: emergency stop); (d) Pictures of moving during ES exercise.

Eccentric contractions were induced at an angular velocity of $30 \% \mathrm{sec}$ as described previously [18]. In this study, we set stimulated time at 1 second. It has been suggested that quadriceps femoris muscle play a crucial role of flexion angle from $0^{\circ}$ to $30^{\circ}$ on walking [20]. Additionally, VI muscle is crucial to the dynamic stability control and may make the greatest contribution to knee extension during dynamic contractions [21] [22]. To stimulate at flexion angle from $0^{\circ}$ to $30^{\circ}$, we set stimulated time at $1 \mathrm{sec}$. The electrical stimulation (carrier frequency: $2500 \mathrm{~Hz}$; and burst modulated frequency: $100 \mathrm{~Hz}$ ) was delivered through a pair of $9 \times 5 \mathrm{~cm}$ gelcoated electrodes attached to the region of the VI muscle belly following described [23].

\subsection{Torque Assessment with ES}

At first, isometric knee extension torque was recorded at MVC using Cybex (CYBEX NORM, CYBEX Division of LUMEX, New York, USA) set at $0 \%$ angular velocity as the subjects sat strapped a chair. Subjects completed 3 maximal isometric repetitions of the dominant limb for $10 \mathrm{sec}$ at $5^{\circ}$ of knee flexion (full knee extension, $0^{\circ}$ ) to match the knee flexion angle of start position. Each maximal isometric repetition was followed by a $3 \mathrm{~min}$ rest interval. During voluntary contractions, participants were encouraged verbally and received visual feedback during each repetition. The greatest peak torque achieved was determined as the maximal voluntary contraction torque. After determined MVC force, the current intensity determined. Current intensity was increased gradually and was determined as the subject's maximum tolerance current level, but no more than $80 \mathrm{~mA}$, with the system start position; the mean value was $49.5 \pm$ 
$5.3 \mathrm{~mA}$. Maximal tolerated intensity was identified as the intensity of stimulation received when the subject said that he could no longer tolerate an increase in intensity. After the intolerance current level set, we set the current intensity induced 30\% MVC force considered comfortable and safety in the present study. The quadriceps muscle torque of maximum voluntary contraction was shown 97 $\pm 9 \mathrm{~N} \cdot \mathrm{m}$. The quadriceps muscle torque of the ES was shown $37 \pm 4 \mathrm{~N} \cdot \mathrm{m}$, and confirmed that the intensity of ES was set 30\% MVC force approximately.

\subsection{Muscle Thickness with Exercise}

While subjects reclined on the training system for the assigned posture with start position, the thickness of the VI muscle was measured with an ultrasound image device with $9 \mathrm{MHz}$ linear transducer (EUB-415, HITACHI medico, Tokyo, Japan) at rest (REST), at MVC, and at stimulated electrically (ES) respectively. Seven healthy untrained men were recruited for the reliability analysis. The intraclass correlation coefficients (ICC) for the test-retest reliability of the muscle thickness measurements were 0.991 (95\% CI 0.971 - 0.996) for the vastus intermedius; these results indicated a high degree of reproducibility in measuring muscle thickness of these muscles.

\subsection{Torque Assessment with Exercise}

In order to evaluate acute response with the developed training system, a dynamometer (GT-30, OG giken, Okayama, Japan), which was incorporated in developed training system as to adhere the front part of the ankle, was used to measure at MVC, during peak flexion torque at with (ES + ECC) and without training system (ES).

\subsection{Pain Evaluation}

To evaluation of pain during using training system, NRS (Numeric Rating Scale) scores was compared between rest condition (REST), ES with (ES + ECC), and without training system (ES). Additionally, to evaluate the subjects intolerance current, NRS was compared between the current intensity was $10 \%$ down from $30 \%$ MVC force (20\% MVC), 30\% MVC (30\% MVC), and $10 \%$ up ( $40 \%$ MVC). For the NRS, the pain intensity was rated on a numerical scale from 0 to $10(0=$ no pain and $10=$ worst pain imaginable). The electrocardiogram (ECG) signals were obtained from a portable ECG recorder (Check My Heart, Daily Care BioMedical, Chungli, Taiwan) and transferred to a computer loaded with heart rate variability (HRV) analysis software. HRV sampling frequency is 250 samples/sec and measured for $5 \mathrm{~min}$. The two components of power of the R-R Interval (RRI: ms.ms), low frequency (LF: $0.04-0.15 \mathrm{~Hz}$ ) and high frequency (HF: 0.15 $0.4 \mathrm{~Hz}$ ), were calculated. The participants were allowed to set supine position comfortably on a training system in a quiet environment for $5 \mathrm{~min}$, as a rest condition. Then, the record of the ECG signal for HRV analysis started. LF/HF ratio was measured at rest (REST), at ES, and at stimulated electrically (ES + 
ECC) respectively. To measure the change of HRV during training, we set the last training period for $5 \mathrm{~min}$.

\subsection{Training Protocol with Electrical Stimulation with Eccentric Contraction Training System}

The previous studies have suggested that it was necessary to induce muscle strengthening at least 50\% MVC [24] [25]. In addition, the pain was depended on current intensity [26]. Therefore, in the long period training trial, the current intensity was set at 50\% MVC with eccentric contraction induced electrical stimulation (current intensity: $36 \pm 7 \mathrm{~mA}$, NRS: $5 \pm 1$ ). The subjects were trained with training system on the right limb and only ES training on the left, three times per week for 6 weeks following the previous study [18]. Pre and post training, subjects were measured thickness of VI muscle and maximum knee extension torque of the both limbs. Before starting first of the training and after 48 $\mathrm{h}$ from last training, subjects reclined supine position and the thickness of the VI muscle was measured with an ultrasound image device with $9 \mathrm{MHz}$ linear transducer. The captured images were measured using the Image J software (NIH, Bethesda, MD, USA). The values pre and post training of the VI muscle were used to calculate the change value of thickness. After measured the thickness of the muscles, isometric knee extension torque was measured at maximum knee extension torque using Cybex (CYBEX NORM, CYBEX Division of LUMEX) set at $0 \%$ sec angular velocity as the subjects sat strapped a chair. Subjects completed maximal isometric repetition of the right and left limbs for $10 \mathrm{sec}$ at $60^{\circ}$ of knee flexion (full knee extension, $0^{\circ}$ ) respectively. Each maximal isometric repetition was followed by a 3 min rest interval. During voluntary contractions, subjects were encouraged verbally and received visual feedback during each repetition. The greatest peak torque achieved was determined as the maximal knee extension torque. The values of pre (right limb: Pre-ES, left limb: Pre-ES + ECC) and post (right limb: Post-ES, left limb: Post-ES + ECC) torques were compared between pre and post, right and left limb, respectively.

\subsection{Data Analysis}

Data were presented as mean $\pm \mathrm{SD}$. In the acute response trial, the thickness of the VI muscle and quadriceps muscle torque, LF/HF measures were obtained for subjects with one-way repeated measures analysis of variance (ANOVA). When a significant difference was found post hoc comparisons were performed using a Bonferroni correction. In the long period training trial of isometric knee extension torque compared pre and post training, differences were assessed by two-way analysis of variance (ANOVA). The Tukey-Kramer post-hoc test was performed if the two-way ANOVA indicated a significant difference. Student's t-test was performed to compare the VI muscle thickness compared pre and post training. Statistical significance was set at $\mathrm{P}<0.05$. To achieve a significant difference at $a$ $=0.05$ and with $80 \%$ power, the necessary and sufficient $\mathrm{n}$ was calculated using 
the mean and SD from a pilot study involving similar experimental groups and from a previous study on the effects of muscle thickness [19].

\section{Results}

\subsection{Experiment 1}

\subsubsection{The Thickness of the Vastus Intermedius Muscle}

The thickness of the VI muscle was thicker in the MVC $(\mathrm{P}<0.05)$ and the ES $(\mathrm{P}$ $<0.05$ ) than in the REST condition (Figure 2). In addition, there were no significant differences between the MVC and the ES.

\subsubsection{The Thickness of the Vastus Intermedius Muscle}

The quadriceps muscle torque of MVC was higher than in the ES and the ES + ECC (Figure 3). Whereas, the quadriceps muscle torque of the ES + ECC was higher than the ES, and was approximately $69 \%$ MVC force.

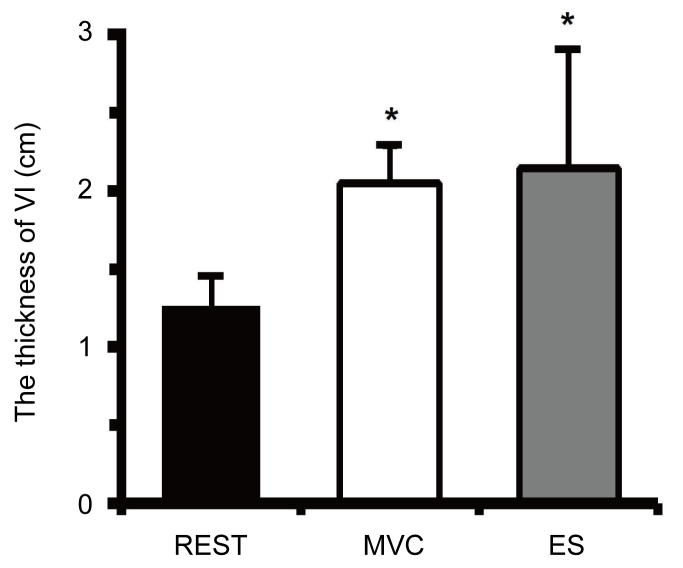

Figure 2. The muscle thickness of vastus intermedius muscle using ultrasound image in the acute response trail at the rest (REST), MVC, and during ES (ES). The thickness is measured and is presented as the mean $\pm \mathrm{SD}$. ${ }^{*}$ indicate significant difference compared to REST at $\mathrm{P}<0.05$.

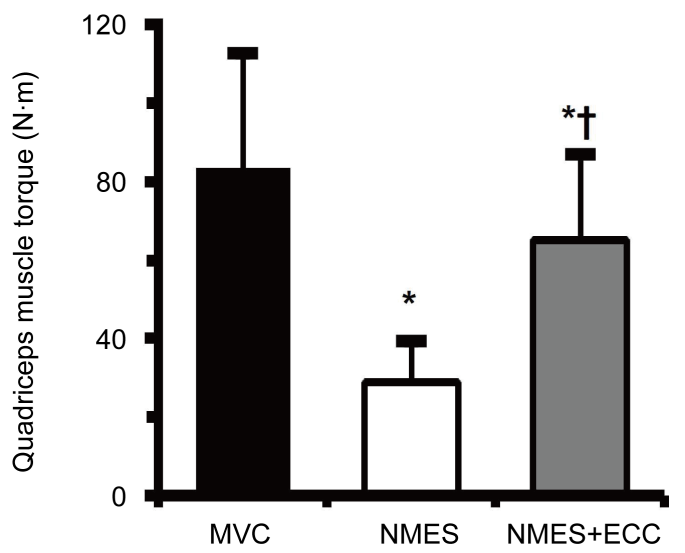

Figure 3. The quadriceps muscle torque with MVC, ES, and eccentric contraction induced ES (ES + ECC) in acute response trial. The quadriceps muscle torque is presented as the mean $\pm \mathrm{SD}$. ${ }^{*}$ and $\dagger$ indicate significant difference compared to MVC and ES, respectively, at $\mathrm{P}<0.05$. 


\subsubsection{Numeric Rating Scale (NRS) Related Increased Current Intensity} The NRS was higher in the $30 \%$ MVC force trial than in the $10 \%$ down trial in which $10 \%$ current down from the current of $30 \%$ MVC force was used, and lower than the $10 \%$ current up trial in which $10 \%$ current up from the current $30 \%$ MVC force was used (Figure 4). Therefore, our results suggested that 30\% MVC force induced the current intensity would be suggested maximum tolerance current level in the present study.

\subsubsection{The Changes in the LF/HF Ratio of HRV}

The LF/HF ratio of HRV in the ES was higher than that in the REST (Figure 5). Whereas, there were no significant differences in the LF/HF ratio of HRV between the ES and the ES + ECC.

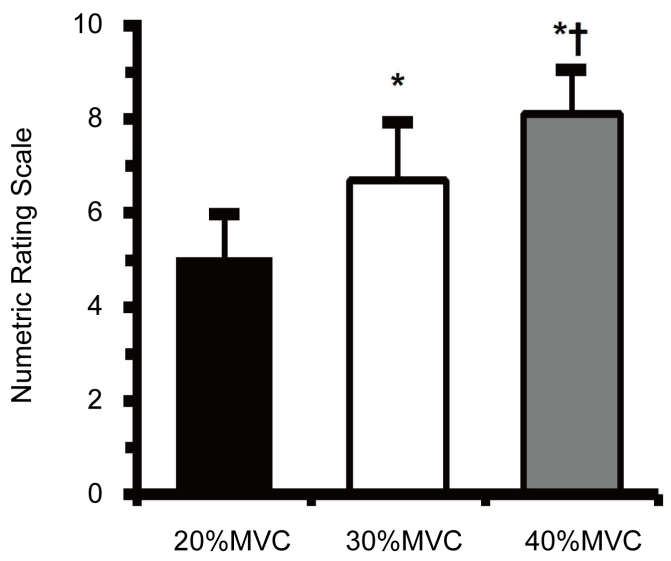

Figure 4. Numetric rating score (NRS) with ES of maximum tolerance current (10\% up), the current induced 30\% MVC force (30\% MVC), and the current 10\% down from $30 \%$ MVC force (10\% down). The subject's maximum tolerance current level was identified as the intensity of stimulation received when the subject said that he could no longer tolerate an increase in intensity. NRS is presented as the mean $\pm \mathrm{SD} .{ }^{*}$ and $\dagger$ indicate significant difference compared to the current $10 \%$ down from $30 \% \mathrm{MVC}$ force and $30 \% \mathrm{MVC}$ force, respectively, at $\mathrm{P}<0.05$.

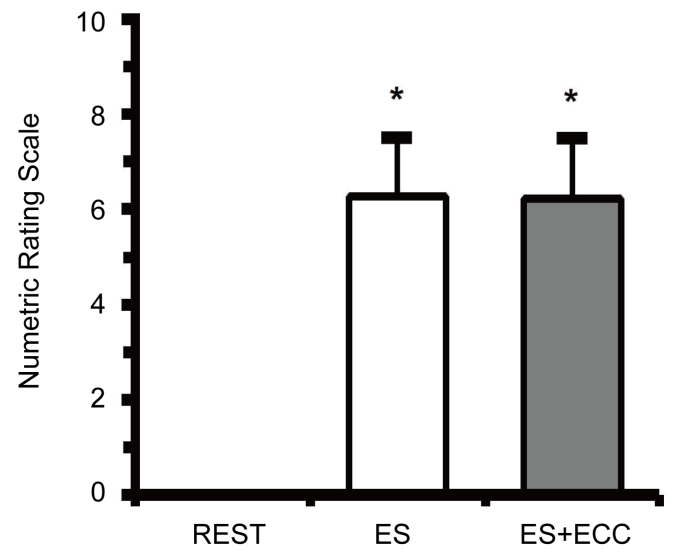

Figure 5. Numetric rating score (NRS) with ES (ES) and eccentric contraction induced $\mathrm{ES}(\mathrm{ES}+\mathrm{ECC})$ and rest condition (REST). NRS is presented as the mean $\pm \mathrm{SD} .{ }^{*}$ indicate significant difference compared to REST at $\mathrm{P}<0.05$. 


\subsubsection{Numeric Rating Scale (NRS) of ES with Eccentric Contraction}

The NRS score in the ES and the ES + ECC was higher than that in the REST (Figure 6). Additionally, there were no significant differences between the ES and the ES + ECC trials.

\subsection{Experiment 2}

The Effects of ES with Eccentric Contraction for Long Period Training The change value of VI muscle thickness in the ES + ECC after 6 weeks training was higher than in the ES (Figure 7). There were no significant differences knee

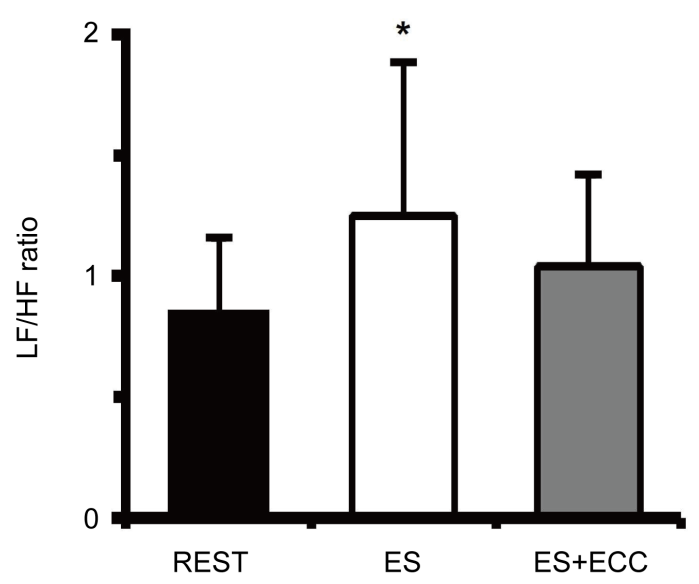

Figure 6. The changes in the LF/HF ratio of HRV at the rest (REST) and during ES (ES) and eccentric contraction induced ES training (ES + ECC). The ECG signal was recorded for heart rate variability (HRV). Frequency fluctuations of HRV were calculated from HRV and identified in the range of $0.04-0.15 \mathrm{~Hz}$ (low frequency, LF) and high frequency (HF) fluctuations in the range of $0.15-0.4 \mathrm{~Hz}$. The LF/HF ratio of $\mathrm{HRV}$ was calculated as the ratio relative the $\mathrm{LF}$ and $\mathrm{HF}$. The $\mathrm{LF} / \mathrm{HF}$ ratio is presented as the mean $\pm \mathrm{SD}$. ${ }^{*}$ indicate significant difference compared to REST at $\mathrm{P}<0.05$.

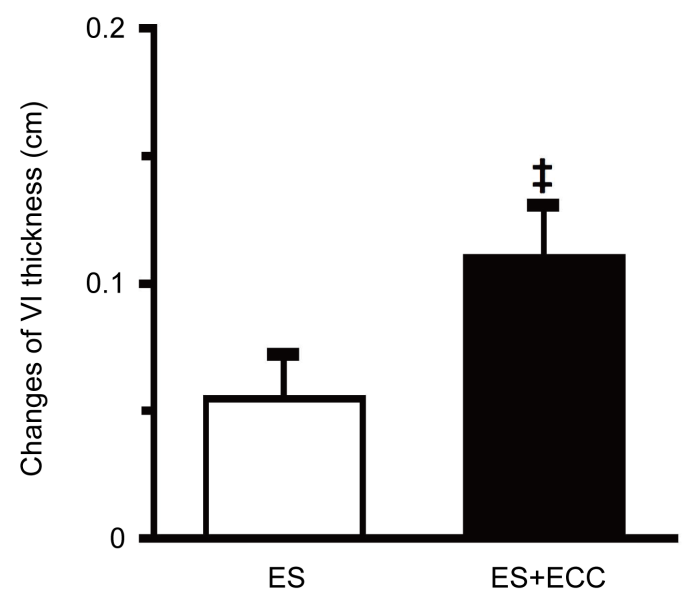

Figure 7. The muscle thickness of vastus intermedius muscle using ultrasound image in the long period training trial. The changed values after training for 6 weeks are shown in the only ES (ES) and ES with eccentric contraction system (ES + ECC). The thickness is measured and is presented as the mean $\pm \mathrm{SD}$. $\ddagger$ indicate significant difference compared to $\mathrm{ES}$ at $\mathrm{P}<0.05$. 
extension torque between Pre-ES and Pre-ES + ECC (Figure 8). However, the knee extension torque of the Post-ES + ECC was higher than the Pre-ES + ECC. Additionally, the Post-ES + ECC was higher than the Post-ES. In contrast, there were no significant differences between the Pre- and the Post-ES.

\section{Discussion}

The main finding of the present study is the promotional effects eccentric contraction training using the training system synchronized ES on the enhancement of loaded muscle torque without enhancing the pain and discomfort induced ES. In addition, eccentric contraction induced ES for 6 weeks training was effective on muscle strengthening. In contrast, the only ES training failed on muscle strengthening. Therefore, our findings suggest that eccentric contraction induced ES might be not only more effective training for muscle strengthening than only ES training, but also available to avoid the increases of pain and discomfort induced by high intensity electrical stimulations which are usually selected to cause strong muscle contraction.

The present study demonstrated the increases of thickness of VI during ES as well as that during MVC in Experiment 1. Recently, ES with middle frequency burst-modulated alternating current has also been used to stimulate skeletal muscles, as well as low frequency direct current [14]. Petrofsky et al. reported that middle frequency alternating current has higher conductivity than low frequency direct current [11]. We have shown that ES with middle frequency burst-modulated alternating current elicited muscle contraction in the deep muscle of rat hindlimb [13]. In the present study, the ES with middle frequency burst-modulated alternating current increased the thickness of VI. Therefore, it is suggested that ES with middle frequency burst-modulated alternating current could be induce effective contraction on deep muscle.

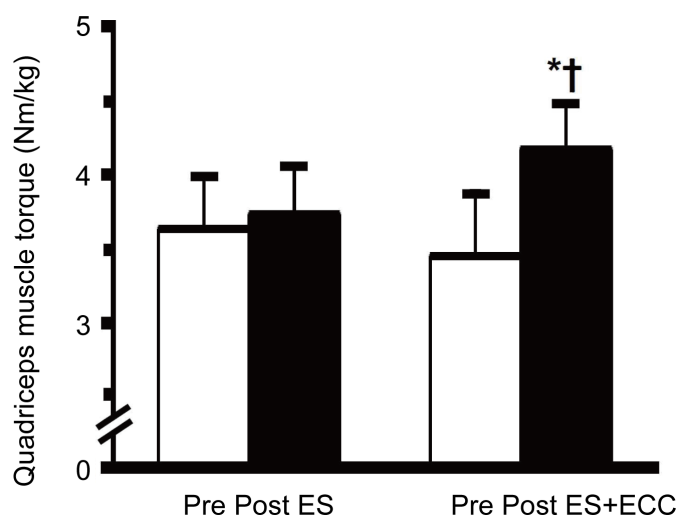

Figure 8. The quadriceps muscle torque ES and eccentric contraction induced ES in the long period training trail. The values of pre (right limb: Pre-ES, left limb: Pre-ES + ECC) and post (right limb: Post-ES, left limb: Post-ES + ECC) torques were compared between pre and post, right and left limb, respectively. The quadriceps muscle torque is presented as the mean \pm SD. ${ }^{*}$ and $\dagger$ indicate significant difference compared to Post-ES and Pre-ES + ECC, respectively, at $\mathrm{P}<0.05$. 
Our results showed that the muscle torque of the ES + ECC was shown approximately $69 \%$ MVC force although that of ES alone was shown 30\% MVC force in the experiment 1 In the present study, we have developed the ES with eccentric contraction system from two points of view. First point was to enhance the promotional effects of muscle strengthening by using eccentric contraction system. The principle of overload is generally recognized as fundamental to the strengthening process, meaning that when the target muscle was loaded with resistance training, the muscle will adapt to become able to enhance the effects of training involving physiological changes e.g. muscle hypertrophy or neural adaptations following increased muscle loading [19] [27]. It has been suggested that eccentric contraction exercise could enhance the loading to target muscle in comparison with isometric and concentric contraction [28]. The results of present study showed that the muscle loading with ES was increased by using eccentric contraction system. Therefore, it has been suggested that ES with eccentric contraction system in this study would be effective for enhancement the effect of ES alone, which lead to muscle strengthening.

Our results (Figure 4) showed that 30\% MVC force was nearly tolerance maximum current intensity for training. However, the muscle loading need at least $50 \%$ MVC force to induce muscle hypertrophy for the healthy subjects [24]. In contrast, muscle loading induced electrical stimulation could also be enhanced by increasing electrical current intensity. However, the increase of pain and discomfort level depends on current intensity during electrical stimulation. It has been reported that some subjects complained severe pain with electrical stimulation for muscle strengthening [29]. This pain could be so uncomfortable that many subjects prefer not using this modality even though there was good therapeutic [29]. Thus, current intensity which was set for ES must be considered a balance between tolerance pain and maximum muscle loading. To suppress increased severe pain and discomfort level was our second point. In the present study, the results of NRS were no significant differences between ES and eccentric contraction induced ES at current intensity of 30\% MVC. Additionally, the results of LF/HF ratio were no significant differences between ES and eccentric contraction induced ES trial. Heart rate variability (HRV) has been used as a biomarker of autonomic nervous system function. HRV is a reliable method to obtain information on sympathetic and parasympathetic contributions to heart rate, and several studies have shown that pain increases sympathetic activity [30] [31]. Frequency fluctuations of HRV in the range of LF are considered to be markers of sympathetic and parasympathetic nerve activity, and HF fluctuations are considered markers of parasympathetic nerve activity [30] [31]. Additionally, the LF/HF ratio is considered an index of sympathetic nerve activity and as an index of pain and discomfort due to activated sympathetic nerve following increased pain and discomfort level [31] [32]. Therefore, in the present study, the results of LF/HF were suggested that the pain and discomfort induced ES could not be enhanced by eccentric contraction induced ES. It has been suggested that nociceptor on the skeletal muscle which was a receptor detected nociceptive sti- 
mulus, e.g. electrical stimulation and muscle stretch located fascia mainly. The nociceptor was related muscle pain and discomfort. In addition, a high-threshold mechanical receptor, which was one of a nociceptor, related the muscle extension [33]. Whereas, high-threshold mechanical receptor detects by muscle to overstretching [34]. In the present study, quadriceps femoris muscle would not be overstretched because the knee angle was moved at flexion angle from $5^{\circ}$ to $30^{\circ}$. Therefore, ES with eccentric contraction system was not enhanced the intensity of pain and discomfort induced by ES.

The results of present study showed that ES with middle frequency could be induced effective muscle contraction on deep muscle, and promotional effect by ES with eccentric contraction system was found in the VI muscle thickness after 6 weeks. In contrast, the only ES training failed on muscle strengthening. The results showed that although ES was induced muscle loading insufficiently for muscle strengthening, ES with eccentric contraction system was induced the muscle loading sufficiently for muscle strengthening at the same time as suppressing increased current intensity. Therefore, it has been suggested that eccentric contraction induced ES would lead to muscle strengthening without sever pain and discomfort even if using only ES induced insufficient muscle loading for muscle strengthening.

The present study has been conducted with limitations. First, the present study was conducted with the healthy men. Therefore, it is unclear that the results of the present study apply the neuromuscular patients, disuse atrophy of the lower limbs of patients, and loss of skeletal muscle mass induced during aging (sarcopenia). Second, the protocol such as current intensity and angular velocity is unknown in effective therapy using eccentric contraction for various patients. Therefore, we plan to perform further studies to answer to the question.

\section{Conclusion}

Eccentric contraction induced ES enhanced muscle torque in the quadriceps femoris muscle in comparison to ES alone. Additionally, eccentric contraction induced ES did not increase pain and discomfort. Moreover, eccentric contraction induced ES for 6 weeks training trial showed to be effective for muscle strengthening. These results suggest that eccentric contraction induced ES would have the potential to become an effective intervention to promote muscle strengthening.

\section{Acknowledgements}

This study was supported by Grants-in-Aid for Scientific Research from the Japanese Ministry of Education, Culture, Sports, Science and Technology, and Japanese Society of Physical Therapy.

\section{Conflict of Interest}

The authors declare that there is no conflict of interest regarding the publication of this article. 


\section{References}

[1] Schoenfeld, B.J. (2010) The Mechanisms of Muscle Hypertrophy and Their Application to Resistance Training. Journal of Strength \& Conditioning Research, 24, 2857-2872. https://doi.org/10.1519/JSC.0b013e3181e840f3

[2] Milne, K.J. and Noble, E.G. (2002) Exercise-Induced Elevation of HSP70 Is Intensity Dependent. Journal of Applied Physiology, 93, 561-568.

https://doi.org/10.1152/japplphysiol.00528.2001

[3] Fitts, R.H. (2003) Effects of Regular Exercise Training on Skeletal Muscle Contractile Function. American Journal of Physical Medicine \& Rehabilitation, 82, 320-331. https://doi.org/10.1097/01.PHM.0000059336.40487.9C

[4] Kirby, C.R., Ryan, M.J. and Booth, F.W. (1992) Eccentric Exercise Training as a Countermeasure to Non-Weight-Bearing Soleus Muscle Atrophy. Journal of Applied Physiology, 73, 1894-1899.

[5] Dudley, G.A., Tesch, P.A., Harris, R.T., Golden, C.L. and Buchanan, P. (1991) Influence of Eccentric Actions on the Metabolic Cost of Resistance Exercise. Aviation, Space, and Environmental Medicine, 62, 678-682.

[6] Colliander, E.B. and Tesch, P.A. (1990) Effects of Eccentric and Concentric Muscle Actions in Resistance Training. Acta Physiologica Scandinavica, 140, 31-39. https://doi.org/10.1111/j.1748-1716.1990.tb08973.x

[7] Fujita, N., Murakami, S. and Fujino, H. (2011) The Combined Effect of Electrical Stimulation and High-Load Isometric Contraction on Protein Degradation Pathways in Muscle Atrophy Induced by Hindlimb Unloading. Journal of Biomedicine and Biotechnology, 2011, Article ID: 401493. https://doi.org/10.1155/2011/401493

[8] Kim, S.J., Roy, R.R., Kim, J.A., Zhong, H., Haddad, F., Baldwin, K.M. and Edgerton, V.R. (2008) Gene Expression during Inactivity-Induced Muscle Atrophy: Effects of Brief Bouts of a Forceful Contraction Countermeasure. Journal of Applied Physiology, 105, 1246-1254. https://doi.org/10.1152/japplphysiol.90668.2008

[9] Kim, S.J., Roy, R.R., Zhong, H., Suzuki, H., Ambartsumyan, L., Haddad, F., Baldwin, K.M. and Edgerton, V.R. (2007) Electromechanical Stimulation Ameliorates Inactivity-Induced Adaptations in the Medial Gastrocnemius of Adult Rats. Journal of Applied Physiology, 103, 195-205.

https://doi.org/10.1152/japplphysiol.01427.2006

[10] Petrofsky, J.S. and Laymon, M. (2002) The Effect of Ageing in Spinal Cord Injured Humans on the Blood Pressure and Heart Rate Responses during Fatiguing Isometric Exercise. European Journal of Applied Physiology, 86, 479-486. https://doi.org/10.1007/s00421-002-0588-2

[11] Petrofsky, J. (2008) The Effect of the Subcutaneous Fat on the Transfer of Current through Skin and into Muscle. Medical Engineering \& Physics, 30, 1168-1176.

[12] Petrofsky, J., Laymon, M., Prowse, M., Gunda, S. and Batt, J. (2009) The Transfer of Current through Skin and Muscle during Electrical Stimulation with Sine, Square, Russian and Interferential Waveforms. Journal of Medical Engineering \& Technology, 33, 170-181. https://doi.org/10.1080/03091900802054580

[13] Tanaka, M., Hirayama, Y., Fujita, N. and Fujino, H. (2013) Comparison of Premodulated Interferential and Pulsed Current Electrical Stimulation in Prevention of Deep Muscle Atrophy in Rats. Journal of Molecular Histology, 44, 203-211. https://doi.org/10.1007/s10735-012-9473-4

[14] Ward, A.R. (2009) Electrical Stimulation Using Kilohertz-Frequency Alternating Current. Physical Therapy, 89, 181-190. https://doi.org/10.2522/ptj.20080060 
[15] Wang, L.C. and Kernell, D. (2001) Quantification of Fibre Type Regionalisation: An Analysis of Lower Hindlimb Muscles in the Rat. Journal of Anatomy, 198, 295-308. https://doi.org/10.1046/j.1469-7580.2001.19830295.x

[16] Ikezoe, T., Mori, N., Nakamura, M. and Ichihashi, N. (2011) Atrophy of the Lower Limbs in Elderly Women: Is It Related to Walking Ability? European Journal of Applied Physiology, 111, 989-995. https://doi.org/10.1007/s00421-010-1728-8

[17] Gregory, C.M. and Bickel, C.S. (2005) Recruitment Patterns in Human Skeletal Muscle during Electrical Stimulation. Physical Therapy, 85, 358-364.

[18] Iwasaki, T., Shiba, N., Matsuse, H., Nago, T., Umezu, Y., Tagawa, Y., Nagata, K. and Basford, J.R. (2006) Improvement in Knee Extension Strength through Training by Means of Combined Electrical Stimulation and Voluntary Muscle Contraction. The Tohoku Journal of Experimental Medicine, 209, 33-40. https://doi.org/10.1620/tjem.209.33

[19] Son, J., Lee, D. and Kim, Y. (2014) Effects of Involuntary Eccentric Contraction Training by Neuromuscular Electrical Stimulation on the Enhancement of Muscle Strength. Clinical Biomechanics, 29, 767-772.

[20] Adler, N., Perry, J., Kent, B. and Robertson, K. (1983) Electromyography of the Vastus medialis Oblique and Vasti in Normal Subjects during Gait. Electroencephalography and Clinical Neurophysiology, 23, 643-639.

[21] Ando, R., Saito, A., Umemura, Y. and Akima, H. (2014) Local Architecture of the Vastus intermedius Is a Better Predictor of Knee Extension Force than That of the Other Quadriceps Femoris Muscle Heads. Clinical Physiology and Functional Imaging, 35, 376-382. https://doi.org/10.1111/cpf.12173

[22] Wang, C.Z., Li, T.J. and Zheng, Y.P. (2014) Shear Modulus Estimation on Vastus Intermedius of Elderly and Young Females over the Entire Range of Isometric Contraction. PLOS ONE, 9, e101769. https://doi.org/10.1371/journal.pone.0101769

[23] Maffiuletti, N.A. (2010) Physiological and Methodological Considerations for the Use of Neuromuscular Electrical Stimulation. European Journal of Applied Physiology, 110, 223-234. https://doi.org/10.1007/s00421-010-1502-y

[24] Filipovic, A., Kleinoder, H., Dormann, U. and Mester, J. (2011) Electromyostimulation-A Systematic Review of the Influence of Training Regimens and Stimulation Parameters on Effectiveness in Electromyostimulation Training of Selected Strength Parameters. The Journal of Strength \& Conditioning Research, 25, 3218-3238. https://doi.org/10.1519/JSC.0b013e318212e3ce

[25] Lyons, C.L., Robb, J.B., Irrgang, J.J. and Fitzgerald, G.K. (2005) Differences in Quadriceps Femoris Muscle Torque When Using a Clinical Electrical Stimulator versus a Portable Electrical Stimulator. Physical Therapy, 85, 44-51.

[26] Han, T.R., Shin, H.I. and Kim, I.S. (2006) Magnetic Stimulation of the Quadriceps Femoris Muscle: Comparison of Pain with Electrical Stimulation. American Journal of Physical Medicine \& Rehabilitation, 85, 593-599. https://doi.org/10.1097/01.phm.0000223239.93539.fe

[27] Hellebrandt, F.A. and Houtz, S.J. (1956) Mechanisms of Muscle Training in Man: Experimental Demonstration of the Overload Principle. Physical Therapy Reviews, $36,371-383$

[28] McCully, K.K. and Faulkner, J.A. (1985) Injury to Skeletal Muscle Fibers of Mice Following Lengthening Contractions. Journal of Applied Physiology, 59, 119-126.

[29] Bennie, S.D., Petrofsky, J.S., Nisperos, J., Tsurudome, M. and Laymon, M. (2002) Toward the Optimal Waveform for Electrical Stimulation of Human Muscle. European Journal of Applied Physiology, 88, 13-19. 
https://doi.org/10.1007/s00421-002-0711-4

[30] Arai, Y.C., Ushida, T., Matsubara, T., Shimo, K., Ito, H., Sato, Y., Wakao, Y. and Komatsu, T. (2011) The Influence of Acupressure at Extra 1 Acupuncture Point on the Spectral Entropy of the EEG and the LF/HF Ratio of Heart Rate Variability. Evidence-Based Complementary and Alternative Medicine, 2011, Article ID: 503698. https://doi.org/10.1093/ecam/nen061

[31] Shiro, Y., Arai, Y.C., Matsubara, T., Isogai, S. and Ushida, T. (2012) Effect of Muscle Load Tasks with Maximal Isometric Contractions on Oxygenation of the Trapezius Muscle and Sympathetic Nervous Activity in Females with Chronic Neck and Shoulder Pain. BMC Musculoskeletal Disorders, 13, 146. https://doi.org/10.1186/1471-2474-13-146

[32] Matsubara, T., Arai, Y.C., Shiro, Y., Shimo, K., Nishihara, M., Sato, J. and Ushida, T. (2011) Comparative Effects of Acupressure at Local and Distal Acupuncture Points on Pain Conditions and Autonomic Function in Females with Chronic Neck Pain. Evidence-Based Complementary and Alternative Medicine, 2011, Article ID: 543291. https://doi.org/10.1155/2011/543291

[33] Stacey, M.J. (1969) Free Nerve Endings in Skeletal Muscle of the Cat. Journal of Anatomy, 105, 231-254.

[34] Mense, S. and Stahnke, M. (1983) Responses in Muscle Afferent Fibres of Slow Conduction Velocity to Contractions and Ischaemia in the Cat. The Journal of Physiology, 342, 383-397. https://doi.org/10.1113/jphysiol.1983.sp014857

\section{Scientific Research Publishing}

Submit or recommend next manuscript to SCIRP and we will provide best service for you:

Accepting pre-submission inquiries through Email, Facebook, LinkedIn, Twitter, etc. A wide selection of journals (inclusive of 9 subjects, more than 200 journals)

Providing 24-hour high-quality service

User-friendly online submission system

Fair and swift peer-review system

Efficient typesetting and proofreading procedure

Display of the result of downloads and visits, as well as the number of cited articles

Maximum dissemination of your research work

Submit your manuscript at: http://papersubmission.scirp.org/

Or contact ijcm@scirp.org 